\title{
CrystEngComm
}

Check for updates

Cite this: CrystEngComm, 2018, 20 1405

Received 30th September 2017, Accepted 2nd January 2018

DOI: 10.1039/c7ce01737e

rsc.li/crystengcomm

\section{Nucleation front instability in two-dimensional (2D) nanosheet gadolinium-doped cerium oxide (CGO) formation}

\author{
Debora Marani, (D)*ab Leticia Poras Reis Moraes, ${ }^{c}$ Fabrizio Gualandris, ${ }^{a}$ \\ Simone Sanna, ${ }^{a}$ Daniel Zanetti de Florio, ${ }^{b}$ \\ Vincenzo Esposito (iD ${ }^{a}$ and Fabio Coral Fonseca (iD ${ }^{c}$
}

\begin{abstract}
Herein we report for the first time the synthesis of ceramic-organic three-dimensional (3D) layered gadolinium-doped cerium oxide $\left(\mathrm{Ce}_{1-x} \mathrm{Gd}_{x} \mathrm{O}_{2-\delta}, \mathrm{CGO}\right)$ and its exfoliation into two-dimensional (2D) nanosheets. We adopt a water-based synthetic route via a homogenous precipitation approach at low temperatures $\left(10-80^{\circ} \mathrm{C}\right)$. The reaction conditions are tuned to investigate the effects of thermal energy on the final morphology. A low temperature $\left(40^{\circ} \mathrm{C}\right)$ morphological transition from nanoparticles (1D) to twodimensional (2D) nanosheets is observed and associated with a low thermal energy transition of ca. $2.6 \mathrm{~kJ}$ $\mathrm{mol}^{-1}$. For the 3D-layered material, exfoliation experiments are conducted in water/ethanol solutions. Systems at volume fractions ranging from 0.15 to 0.35 are demonstrated to promote under ultrasonic treatment the delamination into $2 \mathrm{D}$ nanosheets.
\end{abstract}

\section{Introduction}

In the last few decades, three-dimensional (3D) layered materials have attracted huge interest due to the innumerable possibilities deriving from their peculiar and versatile structure. $^{1-6}$ Such materials consist of stacked twodimensional (2D) nanosheets spaced with interlayer galleries that provide a flexible space to accommodate various sized functional molecules (e.g., pollutants) $)^{2,4,6}$ or specific active sites (e.g. active catalyst materials). ${ }^{2,3,5}$ Layered structures have also attracted interest as a source of $2 \mathrm{D}$ nanosheets, achievable as stable colloidal suspensions via the exfoliation of the 3D structure in an adequate solvent system. ${ }^{7-9} 2 \mathrm{D}$ nanosheets are an emerging class of nanomaterials having atomic thickness (single or a few atomic layers) and infinite planar lengths. ${ }^{10}$ The thickness of the nanoconfinement associated with an extended surface area provide the 2D materials with unique properties compared with their bulk counterparts. ${ }^{9}$ These characteristics have immense potential for a wide range of host applications, ranging from electronic/optoelectronic devices, catalysis, energy storage and conversion, biomedicine, nanoscale sensors, photodetectors and light-

\footnotetext{
${ }^{a}$ Department of Energy Conversion and Storage, Technical University of Denmark (DTU), Frederiksborgvej 399, Roskilde 4000, Denmark.

E-mail: debora.marani3@gmail.com

${ }^{b}$ Centro de Engenharia Modelagem and Ciências Sociais Aplicadas, Universidade Fedearl do ABC, Av. Do Estados 5001, Santo André (SP), 09210-580, Brazil

${ }^{c}$ Nuclear and Energy Research Institute (IPEN-CNEN/SP), 05508-000, São Paulo, SP, Brazil
}

emitting diodes (LEDs). ${ }^{10}$ Given their unique structural features, 2D nanomaterials have also been widely explored as nano-building blocks to fabricate heterostructures. ${ }^{11}$ With this in mind, the exfoliation approach associated with solution-based processing techniques (e.g., inkjet and 3D printing) paves the way for a wide range of innovative materials obtained by adequately re-combining $2 \mathrm{D}$ nanosheets in new 3D structures. ${ }^{12,13}$ This approach enables the design and tailoring of specific properties that can be obtained by combining together different $2 \mathrm{D}$ materials into a single heterostructured material. ${ }^{11}$

However, the solution-based approach poses the challenge of synthesizing the starting 3D layered materials with the desired composition. Several synthetic strategies have been proposed in the literature for the preparation of $3 \mathrm{D}$ layered compounds. The different methods span from solid state reactions ${ }^{14}$ to hydrothermal processes, ${ }^{15}$ including the precipitation approach. ${ }^{16}$ This latter synthetic route has been extensively used in the synthesis of layered double hydroxides (LDHs) as it allows obtaining micrometer-sized and highly crystalline structures. ${ }^{16}$ It is a water-based synthetic route that makes use of urea or hexamethylenetetramine (HMT) as a $\mathrm{pH}$ regulator. ${ }^{17}$ The slow and progressive hydrolysis of urea and HMT makes it possible to attain a homogenous and controlled release of hydroxyl ions in solution. ${ }^{17}$ The reaction likely occurs at the 2D-nucleation front where the layers are formed. Typically, the homogeneous precipitation of layered materials proceeds through the nucleation of positively charged nanosheets followed by their assembly as layered materials via intercalation with negatively charged 
counter anions. This method has been successfully applied to synthesize layered materials other than LDHs, ${ }^{18-21}$ thus enabling other strategic applications. ${ }^{22,23}$

In this work, for the first time, gadolinium-doped cerium (CGO) oxides were synthesized as 3D layered materials via the homogenous precipitation approach at low temperatures $\left(<40{ }^{\circ} \mathrm{C}\right)$. CGO materials were selected because of their relevance in diverse applications, including biomedical ${ }^{24}$ catalysis and environment and energy related technologies. ${ }^{25}$ The use of the water-based homogeneous precipitation route for the preparation of ceria-based oxides has already been reported. ${ }^{19,26,27}$ However, to the best of our knowledge, no investigations about the effect of the reaction conditions on the morphology of the final product have been reported.

The nucleation front for the formation of 2D CGO nanosheets was observed to occur at a relatively low thermal energy $\left(\sim 2.6 \mathrm{~kJ} \mathrm{~mol}^{-1}\right)$. Exfoliation experiments were also successfully conducted using a co-solvent approach. ${ }^{7-9}$

\section{Experimental}

\section{Materials and methods}

All starting chemicals were of reagent grade and stored in a desiccator. Gadolinium nitrate $\left(\mathrm{Gd}\left(\mathrm{NO}_{3}\right)\right.$, Sigma-Aldrich, Brazil) and cerium nitrate $\left(\mathrm{Ce}\left(\mathrm{NO}_{3}\right) \cdot 6 \mathrm{H}_{2} \mathrm{O}\right.$, Sigma-Aldrich, Brazil) were used as precursors to GCO. Hexamethylenetetramine (HMT, Sigma-Aldrich, Brazil) and sodium dodecyl sulfate (SDS, Sigma-Aldrich, Brazil) were used to promote the homogeneous nucleation of the $2 \mathrm{D}$ nanosheets and their precipitation into 3D structures, respectively. Different level of gadolinium doping (e.g. $\mathrm{Gd}_{0.10} \mathrm{Ce}_{0.90} \mathrm{O}_{2}$ and $\mathrm{Gd}_{0.20} \mathrm{Ce}_{0.80} \mathrm{O}_{2}$, indicated as CGO10 and CGO20, respectively) were prepared.

\section{Synthetic procedures}

In a typical procedure, aqueous solutions of cerium nitrate (0.05 M), gadolinium nitrate (0.05 M), SDS (0.1 M), and HMT ( $2 \mathrm{M}$ ) were prepared. The required volume of gadolinium solution (depending on the doping level) was added to $20 \mathrm{ml}$ of cerium nitrate solution. A mixed solution of $10 \mathrm{ml}$ of SDS and $1 \mathrm{ml}$ of HMT was slowly added to the cerium-gadolinium nitrate solution. As the last step, $20 \mathrm{ml}$ of water was added. Different conditions of reaction were studied in the temperature range $80-10{ }^{\circ} \mathrm{C}$. The reactions at 80 and $40{ }^{\circ} \mathrm{C}$ were carried out under reflux and stirring conditions for 6 and 10 hours, respectively. The reactions at low temperatures (30 and $10{ }^{\circ} \mathrm{C}$ ) were conducted in still conditions (no magnetic stirring) for 2 weeks. The reaction time was fixed based on the X-ray diffraction (XRD) results recorded over time. After the reaction, precipitates were filtered and carefully washed with water and ethanol, then dried in a vacuum oven at $30^{\circ} \mathrm{C}$ overnight.

\section{Exfoliation}

The exfoliation reaction was performed in water/ethanol systems with increasing contents of ethanol expressed as vol\% (from 0 to 100 vol\%). In a typical experiment, around $0.5 \mathrm{~g}$ of layered materials were added into $10 \mathrm{ml}$ of the solvent and sonicated at $40{ }^{\circ} \mathrm{C}$ for 8 hours. The solutions were then centrifuged (3000 rpm for $20 \mathrm{~min}$ ) and the supernatants used as nanosheet suspensions.

\section{Characterization}

X-ray diffraction (XRD) was used to characterize both the layered and crystal structures of the materials. A Rigaku MiniFlex (Rigaku Corporation, U.S.A.) diffractometer was used in the $2 \theta$ range of $5-60^{\circ}$. A Rigaku SmartLab (Rigaku Corporation, U.S.A.) diffractometer was used to investigate a larger $\theta-2 \theta$ range $\left(1-90^{\circ}\right)$. Bragg's law, $\lambda=2 d \sin \theta$, was used to calculate the interlayer spacing $d$.

The morphology of the CGO materials was observed by field emission scanning electron microscopy (FESEM, Supra, Carl Zeiss, Germany) and by transmission electron microscopy (TEM, JEM3000F, Oxford Instruments, UK). The chemical composition of the samples was determined by energydispersive spectroscopy (EDS) coupled with FESEM.

\section{Results and discussion}

The influence of temperature on the morphologies was carefully investigated. For all compositions, similar trends in the reaction temperature was observed. As an indication of the temperature-dependent behaviour observed, in Fig. 1a-b, the XRD patterns for CGO10 are reported. A clear effect of the reaction temperature is observed. Consistent with our previous results $^{27}$ and with literature data, ${ }^{26}$ a pure fluorite phase is obtained when the material is prepared at $80^{\circ} \mathrm{C}$.

At decreased temperatures, the typical peaks of a lamellar structure are then identified and associated with the plane $(00 l) .{ }^{28}$ Specific features are observed at different reaction temperatures. Indeed, while the peaks for lamellar and fluorite structures coexist in the XRD pattern of the CGO material prepared at $40{ }^{\circ} \mathrm{C}$, a lamellar structure dominates the material when CGO is prepared at lower temperatures (e.g. 30 and $10^{\circ} \mathrm{C}$ ). It is worth clarifying that in the XRD patterns of CGO prepared at $10^{\circ} \mathrm{C}$ and $30^{\circ} \mathrm{C}$, the presence of the peaks of the lamellar structure might not exclude the presence of the fluorite peaks. The intensity of the peaks of the interlayer planes might have overpowered the fluorite peaks, resulting in their absence in the mean XRD pattern. In contrast, when the synthesized material exhibited no layered structure, the fluorite peaks are observable, and are associated (in the current work) with the CGO nanoparticle formation.

The XRD patterns of the layered material were analysed, and the peaks were assigned to the corresponding diffraction planes (Fig. 1b). The basal spacing for the layered materials was estimated using Bragg's equation using the theta value of the (001) reflection. In Fig. 1b, sharp peaks equidistant from one another suggest highly developed and regular lamellar structures with an $\sim 2.4 \mathrm{~nm}$ spacing, as calculated from the XRD data. Such a relatively large spacing is consistent with the intercalation of the dodecyl sulfate anions (DS 

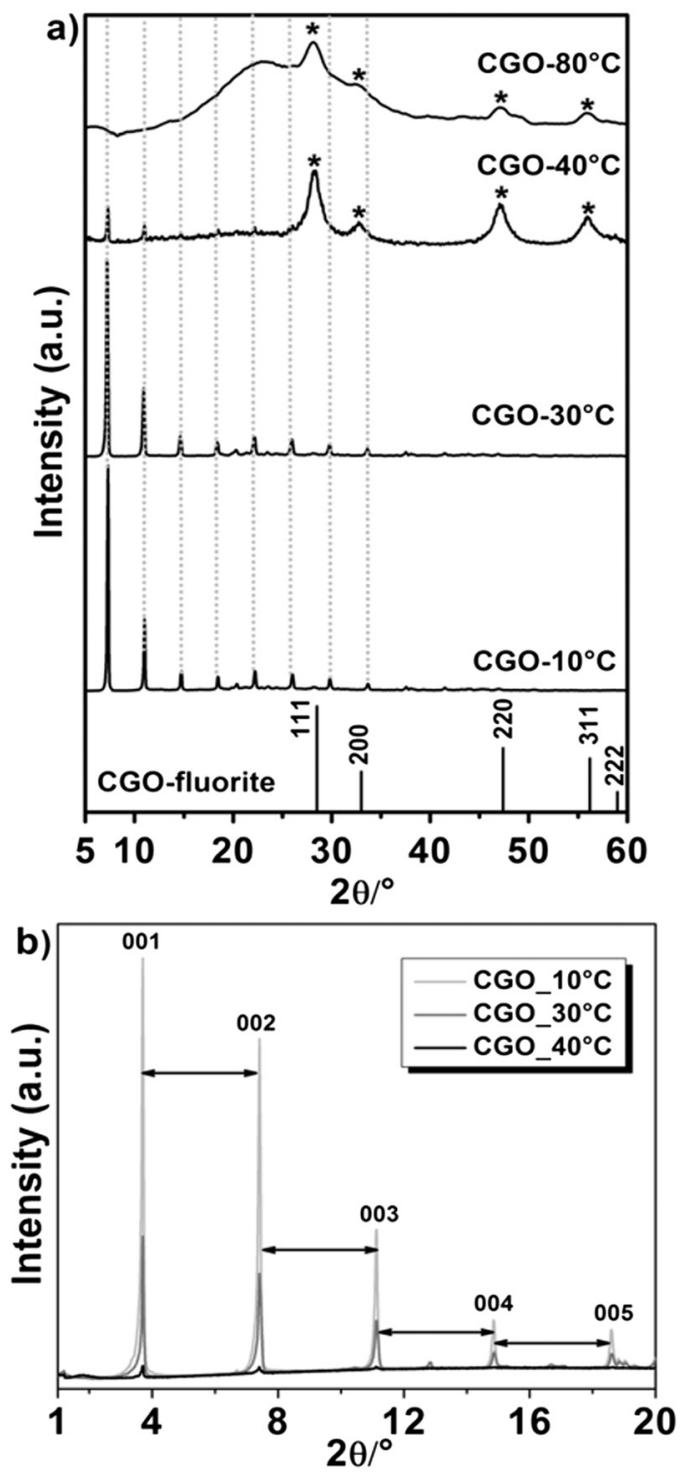

Fig. 1 XRD patterns for the CGO10 materials (a) prepared at different reaction temperatures and (b) for the CGO10 layered materials. The fluorite phase is indicated with asterisks and lamellar planes $(00 l)$ are assigned.

anions $)^{18}$ and it is independent of the reaction temperature (10 and $30^{\circ} \mathrm{C}$ ). Yet, the increment of the peaks intensities with decreasing temperatures is indicative of a corresponding increase in crystallinity.

Fig. 2a-d show the FESEM images for the CGO10 prepared at different temperatures. The FESEM images are reported at different magnifications to emphasize both the morphological features and the effect of the reaction temperature. Fig. 2a shows that the material synthesized at $80{ }^{\circ} \mathrm{C}$ is composed of agglomerated nanoparticles of 5-10 $\mathrm{nm}$. The particles were embedded in a polymeric formation (the dark area in Fig. 2a). This soft chemistry route was found to result in an intense yellow powder with good sintering properties, as reported elsewhere. ${ }^{26,27}$ Fig. $2 \mathrm{~b}$ shows CGO10 synthesized at $40{ }^{\circ} \mathrm{C}$, where a mix of nanoparticles and fibrous layered struc-

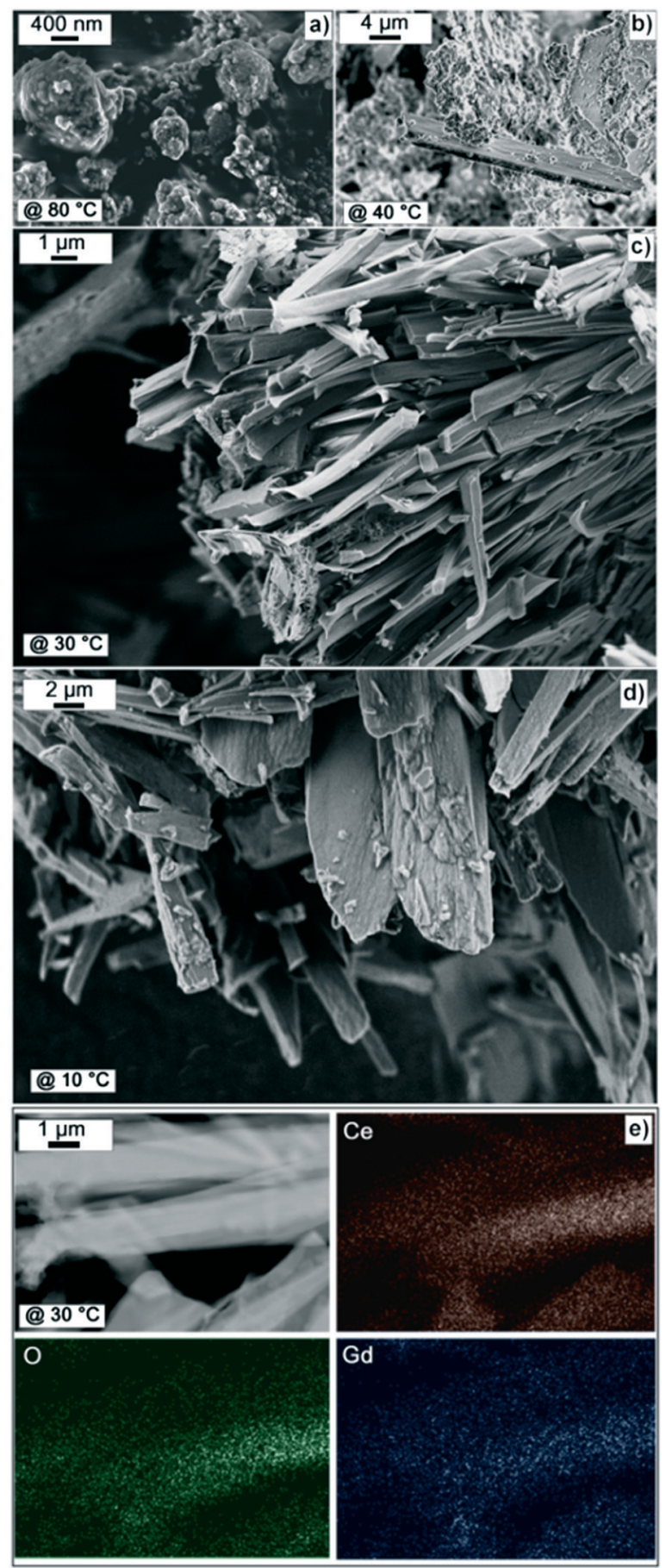

Fig. 2 SEM micrographs for CGO10 samples prepared at (a) $80^{\circ} \mathrm{C}$, (b) $40{ }^{\circ} \mathrm{C}$, (c) $30^{\circ} \mathrm{C}$, and (d) $10^{\circ} \mathrm{C}$; (e) EDS elemental analysis on CGO10 prepared at $30^{\circ} \mathrm{C}$ for cerium (Ce), gadolinium (Gd), and oxygen (O).

tures are observed. Both CGO10 samples synthesized at $30{ }^{\circ} \mathrm{C}$ (Fig. 2c) and $10^{\circ} \mathrm{C}$ (Fig. 2d) possess pure layered morphologies characterized by tubular fibrous structures. A rather homogenous dimensional distribution of the layered structures was observed for the CGO10 prepared at $30{ }^{\circ} \mathrm{C}$. Decreasing the reaction temperature to $10^{\circ} \mathrm{C}$ resulted in larger nanostructures. The CGO materials characterized by a layered morphology (including CGO prepared at $40^{\circ}$ ) exhibited a 
whitish appearance. These results indicate a transition from nanoparticles to $2 \mathrm{D}$ layers controlled by the reaction temperature.

In Fig. 2e, the elemental analysis for CGO10 is reported. A uniform distribution of gadolinium (Gd) is observed, confirming the introduction of Gd into the fluorite structure of cerium oxide $\left(\mathrm{CeO}_{2}\right)$.

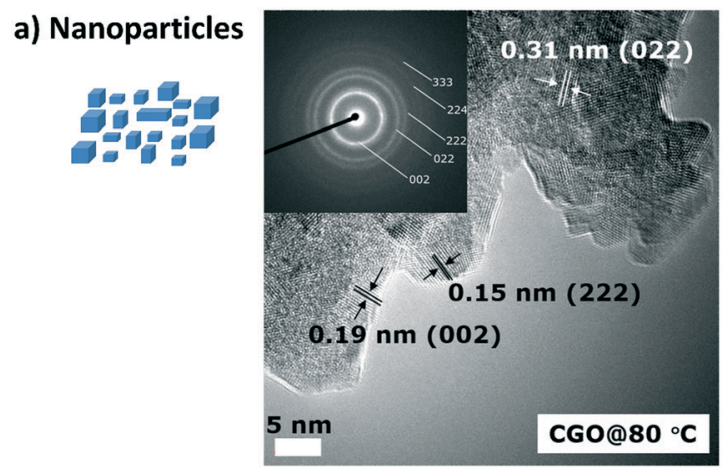

b) Layered structure

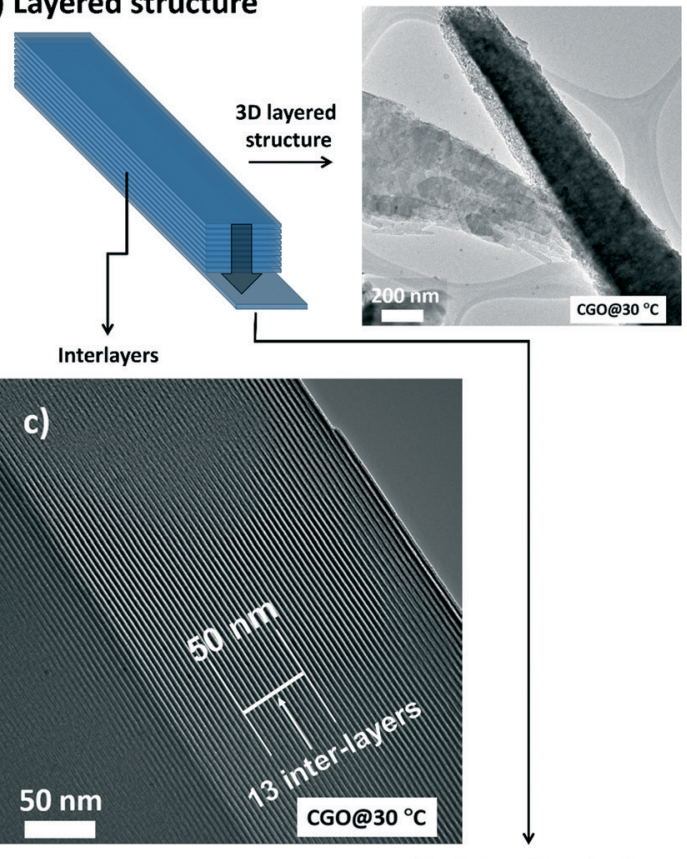

Single layer (top-view)

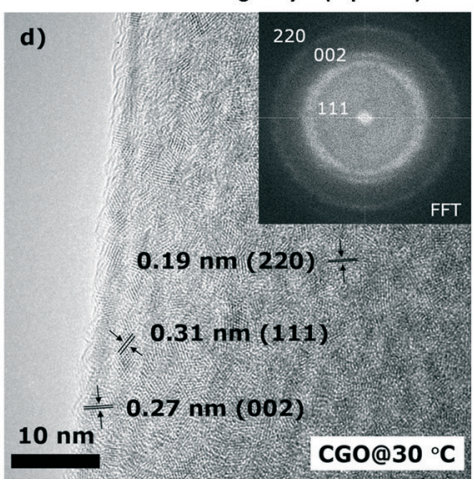

Fig. 3 TEM micrographs for CGO10 prepared at (a) $80^{\circ} \mathrm{C}$ and (b)-(d) 30 ${ }^{\circ} \mathrm{C}$, at different magnifications. The insets in (a) and (d) are SAED patterns.
The morphology and crystallinity of the materials were further investigated by transmission electron microscopy. Fig. 3a shows a TEM micrograph of CGO10 prepared at 80 ${ }^{\circ} \mathrm{C}$.

CGO particles with sizes of around $5 \mathrm{~nm}$ can be observed. The selected area electron diffraction (SAED) pattern, inset in Fig. 3a, suggests the short range (nanoscale) ordering characteristic of the fluorite phase. The tubular structure for the layered materials (CGO10 prepared at $30^{\circ} \mathrm{C}$ ) is confirmed in the TEM micrograph (Fig. 3b). A well-ordered and regular disposition of the lamellas into a $3 \mathrm{D}$ structure is identified (Fig. 3c), with a regular distance from one layer to another of around $4 \mathrm{~nm}$.
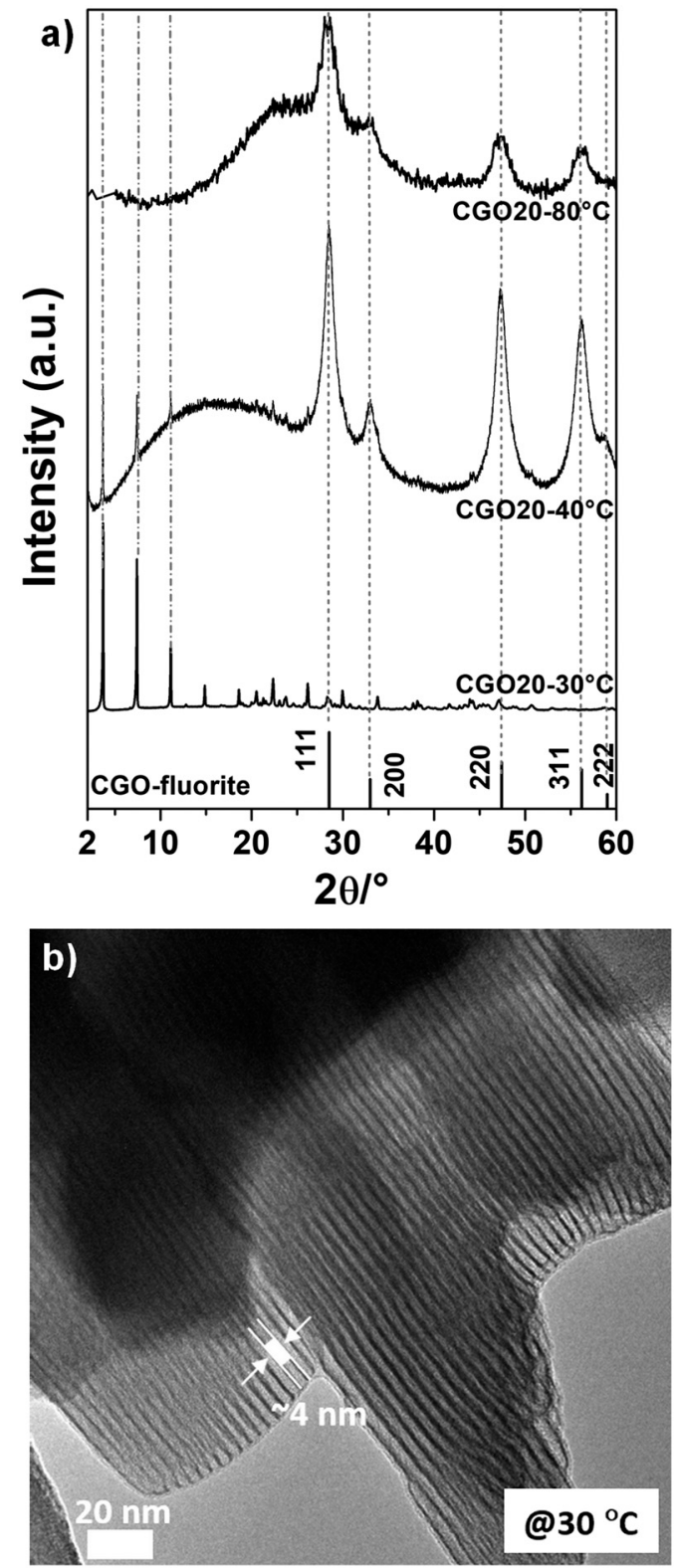

Fig. 4 (a) XRD patterns at different temperatures and (b) TEM micrograph of CGO20. 

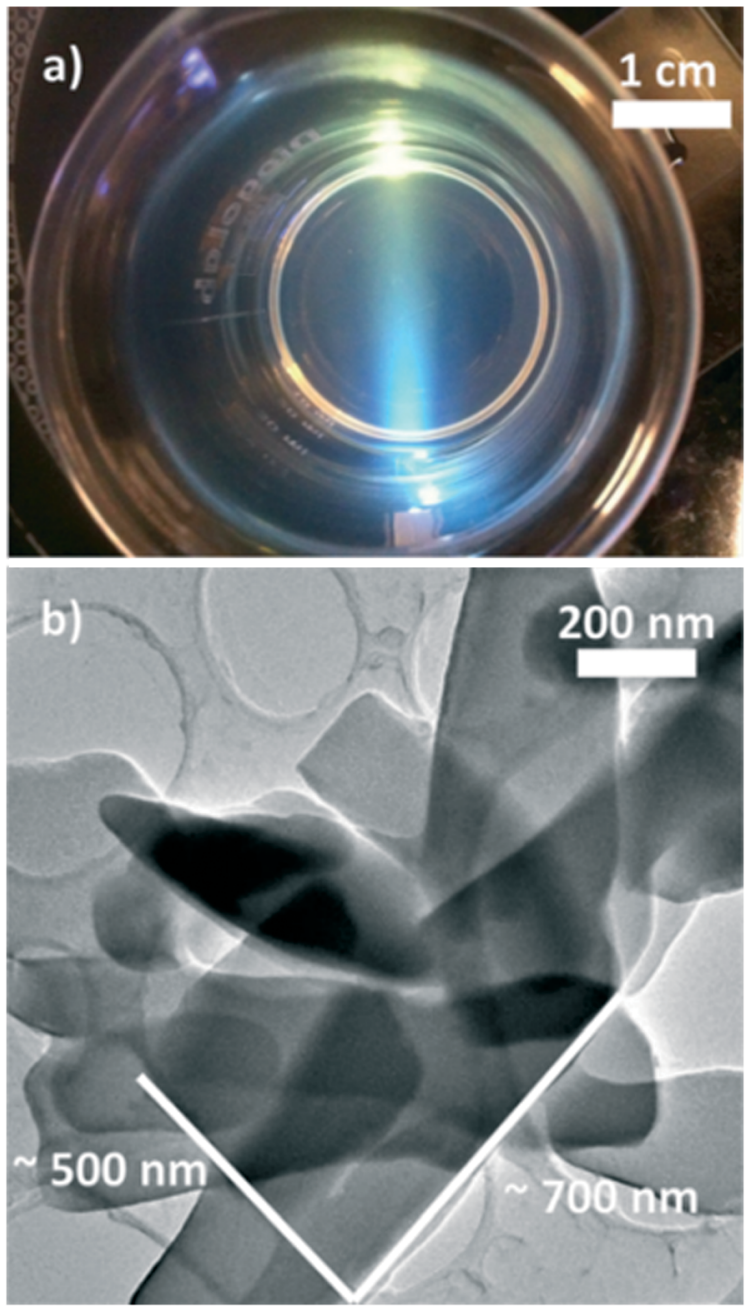

Fig. 5 (a) Photograph of the colloidal suspension of CGO10 exfoliated nanosheets in an ethanol/water co-solvent (at 25 vol\%) system. The light beam was incident from the side to demonstrate the Tyndall effect. (b) TEM micrograph of the CGO nanopackets.

The distance was evaluated by analysing the TEM images. In Fig. 3c, to make it observable, the number of inter-layers in $50 \mathrm{~nm}$ are indicated. The value estimated includes both the thickness of a single CGO nanosheet $(\sim 1 \mathrm{~nm})$ and the thickness of a dodecyl sulfate (DS) anion $(\sim 2.4 \mathrm{~nm}$, as obtained from the XRD pattern). Further, in Fig. 3d, a top view of the $3 \mathrm{D}$ structure indicates that the $2 \mathrm{D}$ stacked layers exhibit a well-defined organization of the atoms. This arrangement corresponds to the fluorite phase, as indicated by the selected-area electron diffraction (SAED) pattern (inset, Fig. 3d) and supported by a fringe spacing of $0.31 \mathrm{~nm}$ indexed to the (111) plane of the fluorite phase.

Electron diffraction (ED) enables us to identify the texture of a single fluorite CGO layer, which was not detected by the XRD analysis.

A dependence of the morphology on the reaction temperature is definitely observed. Such an effect can be reasonably associated with the thermal energy of the system, expressed as $N_{\text {Avogadro }} \times k \times T$. At the lowest temperature, the thermal energy of the system is such as to enable the materials to be arranged into atomically thin 2D nanosheets. At higher values of temperature $\left(\right.$ e.g., $\left.80^{\circ} \mathrm{C}\right)$, the CGO system fails to retain the planar morphology because of the higher thermal energy, and it collapses into nanoparticles (1D). In this 2D1D morphological transition, the threshold temperature is identified to be $40{ }^{\circ} \mathrm{C}$, corresponding to a thermal energy of around $2.6 \mathrm{~kJ} \mathrm{~mol}^{-1}$. At this temperature, both $2 \mathrm{D}$ and $1 \mathrm{D}$ morphologies are observed. This threshold can be associated with a sort of instability for the nucleation front of the $2 \mathrm{D}$ morphology.

Interestingly, regardless of the morphology, all the materials synthesized are polycrystalline with a fluorite arrangement of the atoms (see Fig. 3a and d). This excludes any influence of the growth mechanism of the crystals on the temperature-dependent morphological transition, and it is thus reasonably ascribed to the thermal energy of the system.

No effect of composition (e.g., Gd doping level) was observed either on the 2D-1D morphological transition or on the temperature at which the transition occurred. In Fig. 4a, the XRD patterns for CGO20 obtained at three different reaction temperatures $\left(30^{\circ} \mathrm{C}, 40{ }^{\circ} \mathrm{C}\right.$ and $\left.80^{\circ} \mathrm{C}\right)$ are reported. As already pointed out for CGO10, at increasing temperatures a clear transition from a pattern originating from the lamellar structures to the typical peaks of the fluorite phase (that identifies the nanoparticles) is observed. Again, at $40{ }^{\circ} \mathrm{C}$, a mix of lamellar and fluorite peaks is detected, suggesting this value of temperature as a threshold in the 2D-1D transition. For the layered materials, an interlayer distance of around $2.4 \mathrm{~nm}$ is obtained, which is in agreement with the interlayer distance estimated for CGO10. The TEM micrographs for the CGO20 materials confirm both the nature of the nanoparticles and the lamellar structures for the products prepared at temperatures higher and lower than $40{ }^{\circ} \mathrm{C}$, respectively (not shown). For brevity, herein, only the TEM micrograph of CGO20 prepared at $30{ }^{\circ} \mathrm{C}$ is shown (see Fig. 4b). A well-ordered lamellar structure is definitely observed with an average distance of around $4 \mathrm{~nm}$, which is in agreement with the interlayer distance obtained for the CGO10 materials with the dodecyl sulfate anion intercalation.

Preliminary exfoliation experiments were carried out by sonicating the CGO10 layered structures prepared at $30^{\circ} \mathrm{C}$ in a water/ethanol solvent. A number of publications have demonstrated that an efficient exfoliation process is only achieved by using solvents that can minimize the interfacial energetic cost. This is usually obtained when the surface tension of the solvent matches the energy associated with the 3D structures (e.g. the energy required to exfoliate the layered structure). ${ }^{7,29-31}$ The typical translucent aspect and the Tyndall effect, characteristic of nanosheet colloidal suspensions, ${ }^{29,32,33}$ were observed for the solutions at water/ethanol volume ratios varying from 0.15 to 0.35 (see Fig. $5 b$ ).

Fig. $5 \mathrm{~b}$ shows a TEM micrograph of the CGO nanosheets dispersed in the colloidal solution. Lamellar structures characterized by lateral dimensions of hundreds of nanometres 
are observed. It is worth specifying that the thin nanosheets obtained are likely nanopackets containing a small number $(<10)$ of stacked monolayers, as previously reported (Fig. 5). ${ }^{32,33}$

\section{Conclusions}

For the first-time, CGO layered materials were synthesized and exfoliated into 2D CGO nanosheets. A water-based homogeneous precipitation route was adopted at a temperature $<40{ }^{\circ} \mathrm{C}$. A low energetic threshold for the formation of the 2D layers was identified ( $\mathrm{ca} .2 .6 \mathrm{~kJ} \mathrm{~mol}^{-1}, 40{ }^{\circ} \mathrm{C}$ ), suggesting thermodynamic control over the morphology of the final product. The exfoliation into $2 \mathrm{D}$ nanosheets was obtained in water/ethanol solvent systems at volume ratios ranging from 0.15 to 0.35 .

\section{Conflicts of interest}

There are no conflicts to declare.

\section{Acknowledgements}

The authors gratefully acknowledge the financial support of the Brazilian agencies FAPESP (2014/09087-4, 2014/50279-4, and 2015/20434-0) and CNPq (401218-2014-7 and 311803/20156). Daniel Zanetti de Florio, Fabio Coral Fonseca, and Leticia Poras Reis de Moraes are CNPq fellows. The authors also gratefully acknowledge the Danish Council for Independent Research | Technology and Production Sciences for the DFF-Research Project 2 (grant no. 48293) which financially supported the activity of Vincenzo Esposito. The authors gratefully acknowledge Dr. Paul Tanchida for the revision of the English in this manuscript.

\section{Notes and references}

1 J.-M. Oh, T. T. Biswick and J.-H. Choy, J. Mater. Chem., 2009, 19, 2553.

2 C. Li, M. Wei, D. G. Evans and X. Duan, Small, 2014, 10, 4469.

3 G. Fan, F. Li, D. G. Evans and X. Duan, Chem. Soc. Rev., 2014, 43, 7040.

4 N. Chubar, R. Gilmour, V. Gerda, M. Mičušik, M. Omastova, K. Heister, P. Man, J. Fraissard and V. Zaitsev, Adv. Colloid Interface Sci., 2017, 245, 62.

5 S. He, Z. An, M. Wei, D. G. Evans and X. Duan, Chem. Commun., 2013, 49, 5912.

6 K. Kalantar-Zadeh, J. Z. Ou, T. Daeneke, M. S. Strano, M. Pumera and S. L. Gras, Adv. Funct. Mater., 2015, 25, 5086.

7 V. Nicolosi, M. Chhowalla, M. G. Kanatzidis, M. S. Strano and J. N. Coleman, Science, 2013, 340, 1226419.
8 R. Ma and T. Sasaki, Adv. Mater., 2010, 22, 5082.

9 H. Zhang, ACS Nano, 2015, 9, 9451.

10 C. Tan, X. Cao, X. J. Wu, Q. He, J. Yang, X. Zhang, J. Chen, W. Zhao, S. Han, G. H. Nam, M. Sindor and H. Zhang, Chem. Rev., 2017, 10, 6225.

11 E. Pomerantseva and Y. Gogotsi, Nat. Energy, 2017, 2, 17089.

12 X. Long, Z. Wang, S. Xiao, Y. An and S. Yang, Mater. Today, 2016, 19, 213.

13 J. E. ten Elsof, H. Yuan and P. G. Rodriguez, Adv. Energy Mater., 2016, 6, 1600355.

14 T. Zaremba, J. Therm. Anal. Calorim., 2008, 91, 911.

15 J. S. Chen, M. F. Ng, H. B. Wu, L. Zhang and X. W. Lou, CrystEngComm, 2012, 14, 5133.

16 Z. Liu, R. Ma, M. Osada, N. Ivy, Y. Ebina, K. Takada and T. Sasaki, J. Am. Chem. Soc., 2006, 128, 4872.

17 J. Tao, W. Jiang, H. Pan, X. Xu and R. Tang, J. Cryst. Growth, 2007, 308, 151.

18 S. Ida, D. Shiga, M. Koinuma and Y. Mtsumoto, J. Am. Chem. Soc., 2008, 130, 14038.

19 T. Taniguchi, Y. Sonada, M. Echikawa, Y. Watanabe, K. Hatakeyama, S. Ida and M. Koinuma, ACS Appl. Mater. Interfaces, 2012, 4, 1010.

20 A. Funatsu, M. Koinuma, T. Taniguchi, K. Hatakeyama, Y. Okazawa, Y. Fukunaga, H. Tateishi, C. Ogata and Y. Matsumoto, RSC Adv., 2013, 3, 21343.

21 A. Funatsu, H. Tateishi, K. Hatakeyama, Y. Fukunaga, T. Taniguchi, M. Koinuma, H. Matsuura and Y. Matsumoto, Chem. Commun., 2014, 50, 8503.

22 X. Zhang and Y. Xie, Chem. Soc. Rev., 2013, 42, 8187.

23 C. Tan and H. Zhang, Nat. Commun., 2015, 6, 7873.

24 F. Caputo, M. Mameli, A. Sienkiewicz, S. Licoccia, F. Stellacci, L. Ghibelli and E. Traversa, Sci. Rep., 2017, 7, 4636.

25 C. Sun, H. Li and L. Chen, Energy Environ. Sci., 2012, 5, 8475.

26 P. L. Chen and I. W. Chen, J. Am. Ceram. Soc., 1993, 76, 1577.

27 M. F. S. Machado, L. P. R. Moraes, N. K. Monteiro, V. Esposito, D. Z. de Florio, D. Marani and F. C. Fonseca, ECS Trans., 2017, 78, 387.

28 P. C. Junk, C. J. Kepert, B. W. Skelton and A. H. White, Aust. J. Chem., 1999, 52, 601.

29 H. Usui, T. Sasaki and N. Koshizaki, Appl. Phys. Lett., 2005, 87, 063105.

30 R. Ma, Z. Liu, L. Li, N. Ivy and T. Sasaki, J. Mater. Chem., 2006, 16, 3809.

31 U. Halim, C. R. Zheng, Y. Chen, Z. Lin, S. Jiang, R. Cheng, Y. Huang and X. Duan, Nat. Commun., 2013, 4, 2213.

32 D. Marani, S. Licoccia, E. Traversa and M. Miyayama, Key Eng. Mater., 2010, 421-422, 447.

33 D. Marani, A. D'Epifanio, E. Traversa, M. Miyayama and S. Licoccia, Chem. Mater., 2010, 22, 1126. 